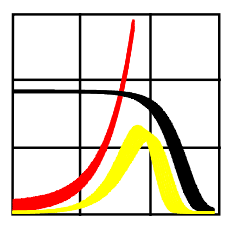

Max-Planck-Institut für demografische Forschung

Max Planck Institute for Demographic Research

Doberaner Strasse 114 - D-18057 Rostock · GERMANY

Tel +49 (0) 3812081 - 0; Fax +49 (0) 3812081 - 202;

http://www.demogr.mpg.de

MPIDR WORKING PAPER WP 1999-014

NOVEMBER 1999

\title{
Macro-Demographic Effects of the
}

Transition to Adulthood: Multistate

Stable Population Theory and an

Application to Italy

Francesco C. Billari (billari@ demogr.mpg.de)

Piero Manfredi (manfredi@ec.unipi.it)

Alessandro Valentini (valentini@ec.unipi.it)

(C) Copyright is held by the authors.

Working papers of the Max Planck Institute for Demographic Research receive only limited review. Views or opinions expressed in working papers are attributable to the authors and do not necessarily reflect those of the Institute. 
Macro-Demographic Effects of the Transition to Adulthood:

\section{Multistate Stable Population Theory and an Application to Italy}

(Running head: Macro-Demographic Effects of the Transition to Adulthood)

by

Francesco C. Billari

Max Planck Institute for Demographic Research

Doberaner Str. 114

D-18057 Rostock

Germany

E-mail: billari@demogr.mpg.de

(corresponding author)

Piero Manfredi and Alessandro Valentini

Dipartimento di Statistica e Matematica Applicata all’Economia

Università di Pisa

Via Ridofi 10

I-56124 Pisa

Italy

E-mail:manfredi@ec.unipi.it, valentini@ec.unipi.it 


\title{
Macro-Demographic Effects of the Transition to Adulthood: Multistate Stable Population Theory and an Application to Italy
}

Running head:

Macro-Demographic Effects of the Transition to Adulthood

\begin{abstract}
We exploit a multistate generalisation of a classical, one-sex, stable population model to evaluate structural and long-term effects of changes in the attainment of adulthood. The demographic framework that inspired this paper is provided by Italy, where a strong delay in the transition to adulthood and union formation has been observed over the last several decades. Italy has also experienced very low fertility levels, and the subsequent ageing problems have become of primary concern. We first discuss a theoretical framework based on the model developed by Inaba (1996) and then include the process of transition to adulthood. We consider explicitly some specifications of the general model, and we present two distinct empirical applications, one using macrosimulation and the other one using a linear approximation. Our principal aim is to evaluate the impact of the delay in the attainment of adulthood on reproduction and on the age structure of the population.
\end{abstract}

$$
\text { (150 words) }
$$

Keywords: stable population theory, multistate demography, transition to adulthood, population ageing, macrosimulation, Italy 


\section{INTRODUCTION ${ }^{1}$}

In this paper, we start from two different points. The first one is a theoretical need: we want to assess the use of classical mathematical demography models, such as a stable population in a multistate framework, in order to evaluate the macro-demographic impact of changes in microlevel behaviour. The second starting point is an explanatory necessity which arises from the observation of demographic behaviour in Italy during the last twenty years. We try to answer one of the open research questions in the transition to adulthood and population dynamics: to outline the 'macro' consequences of changes in the process of transition to adulthood for the overall evolution of a population ${ }^{2}$.

We shall discuss these two points in a little bit more in depth in the following part of this introduction. In Section 2, we set up the mathematical framework of a multistate model that embeds the process of transition to adulthood. The model will be used to address the questions outlined in the introduction. In Section 3, some selected sub-cases of our model will be developed analytically. Section 4 is an introduction to the empirical applications of the model: we discuss the interesting case of Italy, using both survey and official data. A macrosimulation perspective will be adopted for the application in Section 5: there, we will evaluate the effect of delayed adulthood attainment on the dynamics of the population as a whole. A simple linear relationship will be used in Section six to evaluate the impact of delayed transition to adulthood on the overall fertility level. Finally, we provide some conclusions and outline directions for future research.

\footnotetext{
${ }^{1}$ Earlier versions of this paper and a related one were presented at the workshop on Synthetic Biographies, S. Miniato (Italy), 6-9 June 1999, and at the session 'Explaining population dynamics: the use of theories' at the European Population Conference, The Hague (The Netherlands), 30 August-4 September 1999. The authors warmly thank the participants for several useful comments and questions, especially Herwig Birg, Jan Hoem, Alexia Fürnkranz-Prskawetz, Evert Van Imhoff, Zeng Yi and Karl Brehmer (for language editing). The usual caveats apply. The views expressed in this paper are the authors' own views amd do not necessarily represet those of the Max Planck Institute for Demographic Research or the University of Pisa.

${ }^{2}$ The importance of the issue is emphasised - with reference to the American case - by Marini (1984).
} 
1.1. Is there any room left for stable-population theory in the demography of western countries?

Let us give an example of a research question central to our purposes, which we will develop later in the paper: what are the consequences of a postponement in the process of transition to adulthood on the overall fertility level? And what are the consequences on the long-term age structure of the population and, indirectly, on the equilibrium of a pay-as-you-go pension system? How can we organise a technical toolkit to answer such questions?

Event history analysis, which has been used extensively in population studies in the last several years, laid the foundations for an effective empirical study of demographic behaviour at the micro level. When one wishes to evaluate the impact of aggregate factors and of individual demographic behaviour on the experience of an individual (that is, how the macro and the micro affect the micro), such techniques are certainly the best that can be adopted. In contrast, it is difficult to use such approaches when one wishes to outline the emergence of macro-effects of changes in micro-level behaviour, i.e., when one wants to "close the bathtub" of social theory (see Coleman, 1990). Moreover, new micro approaches seem to have almost no connection with something that was at the heart of demography some decades ago: the study of population dynamics. In other words, by using event history analysis only, it is difficult to evaluate how individual behaviours have an impact on the macro-level - a level that was central to classical stable population modelling.

It has been stressed elsewhere (see e.g. Gilbert and Troitzsch, 1999) that simulation is the main tool for providing answers to complex questions on social systems, especially when one is interested on the effects of the behaviour of single interacting agents. We, too, are of this opinion. Approaches such as macrosimulation, microsimulation, or artificial agents may provide fundamental keys for answering questions such as the ones just mentioned. However, we feel that simulation could be even more informative if accompanied by a more analytically oriented approach. In our case, the mathematical toolkit we have is the well-known stablepopulation theory, in its multistate generalisation. Here, while the parameters of the dynamics 
of stable-population theory may represent the micro - though in a very simple way (synthesising the behaviour of the individuals belonging to the population we are interested in) - the outcomes of the models may shed important light on the macro. This is the perspective we adopt in this paper, the theoretical side of which owes much inspiration to Inaba (1996). It is also necessary to state the following precisely: when it comes to empirical applications based on real data, there exist no substantial boundaries between stable-population theory and macrosimulation. In fact, one of the straightforward empirical implementations of the mathematical model we are developing is via macrosimulation.

1.2. The Italian demographic scenario: delayed adulthood attainment and union formation, lower fertility

Let us now sketch out the specific demographic setting we have in mind. In some Western European countries in the Mediterranean region, of which Italy is a particularly noteworthy example, the process of family formation, which takes place generally within a legal marriage, has significantly slowed during the last decades. The transition to adulthood is also characterised there by a strong synchronisation between marriage ${ }^{3}$ and leaving the parental home. Moreover, the length of full-time education for young adults has been steadily increasing, especially for women.

Some studies on the process of transition to adulthood suggest that there are typical sequences between events in early adulthood (e.g. Corijn, 1996), even if it is unclear what the impact of aggregation on such ideas is (Billari, 1999). In any case, having left full-time education - or at least having left the parental home - seems to be a necessary condition for entering a steady (married or unmarried) cohabiting partnership. Then, being in a steady cohabiting partnership seems to be an almost necessary condition for becoming a parent, and this is particularly true in

\footnotetext{
${ }^{3}$ In this paper we will use the words "marriage" and "union" interchangeably, and "marriageable" will be used in the sense "willing to enter any union". The low cohabitation intensity in Italy justifies this approach. However, the focus of the model is in fact on unions.
} 
Southern European countries. It is of great interest, therefore, to evaluate the long-term and macro impacts of changes in adulthood attainment in this context.

Here, we adopt the hypothesis that there is a main 'marker' in the adulthood attainment process that gives rise to the passage to what we can call the 'marriageable' state - following the seminal ideas of Coale and McNeil (1972) - or, less specifically, the 'adult' state. In general, we might say that there is a main marker distinguishing young people (by that we mean people that do not consider entering a union as an option) from adults (people who consider entering a union as an option, or who have already been in a union). Coale and McNeil state that "in contemporary populations of Western European origin (...) we may conjecture that the age of becoming marriageable is the age at which serious dating, or going steady begins".

In this paper we take a specific event as the empirical marker of the transition to adulthood: namely, the first occurring event between the end of formal education and the leaving of the parental home. Adulthood attainment is considered to be irreversible. This is in the spirit of what has been called the Southern European pattern (Reher, 1998). We provide here some justification for the choice of markers. Formal education seems to us a sensible marker because: 1) sooner or later education will be completed, which means that we may assume a unity quantum and just study the tempo of the event;

2) as Blossfeld and De Rose state about Italy, "finishing education is expected to count as one of the important prerequisites for entering into adulthood status, and thereby entering into marriage and parenthood"4.

In addition, when people leave the parental home before the completion of full-time education, we may say that in a sense they may become more ready for unions (and we mainly here refer to informal unions).

\footnotetext{
${ }^{4}$ A framework where the end of formal education is a necessary condition for entering a 'marriageable' state may also well apply to developing countries.
} 


\section{THE THEORETICAL FRAMEWORK: A MULTISTATE STABLE POPULATION}

\section{MODEL WITH AN “ADULT” STATE}

Our theoretical framework is represented by a one-sex, four-state stable population model with irreversible transitions ${ }^{5}$, which generalizes Inaba's (1996) model with reproduction via first marriage. Multistate stable population models arose in demography as further developments of the classical Rogers' multiregional model (see Rogers, 1975). Instances of previous applications of such models in demography are Schoen (1988), Inaba (1988), Ledent (1988), and Rogers (1990). As represented in the flow diagram (Fig. 1), young individuals cannot marry, since they do not become 'marriageable' until they become adults. Moreover only married individuals reproduce. This scheme, as we mentioned, is most apt to represent the patterns of Southern European countries.

\section{[Figure 1 about here]}

This section is mainly theoretical: we first introduce (subsection 2.1) our general model, which recognises both the chronological age and the duration of permanence in each state, and report some basic results concerning its stable state. Subsequently (section 2.2) we show how the formulas for the reproduction indices in the presence of multiple duration-dependencies, extend to the present case.

2.1 Formulation of the general model with multiple duration-dependencies and its long-term solution: stable distributions with respect to age and stages of life

Let $p_{0}(a, t)$ denote the density of 'young' (female) individuals aged a at time t, $p_{I}(c, t ; \eta)$ the density at time $\mathrm{t}$ of adult individuals who have entered into adulthood in the last $c$ years at the age of $\eta, p_{2}(\tau, t ; \eta, \xi)$, the density of individuals married since $\tau$ years who became adult at the age of $\eta$ and married at the age of $\xi$, and $p_{3}(a, t)$ the density of individuals who are not in the 
first three states (widowed, divorced, or remarried). Moreover, let $\mu(a)$ denote the agedependent mortality rate (or force of mortality), $v(a)$ the rate of transition to adulthood, $\lambda(a, \eta)=$ $\lambda(c+\eta, \eta)$ the force of first marriage, which is assumed to be influenced by the age of entrance into adulthood, and $\delta(\tau, \xi)$ the force of dissolution of pairs married $\tau$ years before the female was aged $\xi$.

The backbone of the model is given by the following system of Von Foerster equations:

$$
\left\{\begin{array}{l}
\Delta_{a, t} p_{0}(a, t)=-[\mu(a)+v(a)] p_{0}(a, t) \\
\Delta_{c, t} p_{1}(c, t ; \eta)=-[\mu(c+\eta)+\lambda(c+\eta, \eta)] p_{1}(c, t, \eta) \\
\Delta_{\tau, t} p_{2}(\tau, t ; \eta, \xi)=-[\mu(\xi+\tau)+\delta(\tau, \xi)] p_{2}(\tau, t ; \eta, \xi) \\
\Delta_{a, t} p_{3}(a, t)=\iint_{\tau+\xi=a} \delta(\tau, \xi) p_{2}(\tau, t ; \eta, \xi) d \tau d \eta-\mu(a) p_{3}(a, t)
\end{array}\right.
$$

where $\Delta_{a, t}$ is a shortcut for the aging operator $\frac{\partial}{\partial a}+\frac{\partial}{\partial t}$ and similarly $\Delta_{c, t}, \Delta_{\tau, t}$. The system (1) has to be completed with the boundary conditions:

$$
\left\{\begin{array}{l}
p_{0}(0, t)=B(t)=\int_{0}^{\beta} \int_{\eta}^{\beta} \int_{0}^{\beta-\xi} p_{2}(\tau, t ; \xi, \eta) m(\tau ; \xi, \eta) d \tau d \xi d \eta \\
p_{1}(0, t ; \eta)=v(\eta) p_{0}(\eta, t) \\
p_{2}(0, t ; \eta, \xi)=\lambda(\xi, \eta) p_{1}(\xi-\eta, t ; \eta) \\
p_{3}(0, t)=0
\end{array}\right.
$$

The conditions (2) state, respectively, that:

i) the number of individuals aged zero in the first state at time $t$ is simply the number $B(t)$ of births at time $t, \beta$ is the upper bound of the fertile age span, and $m(\tau ; \xi, \eta)$ the marital fertility rate at marriage duration $\tau$ for a married women who entered the adult state at age $\eta$ and then married at age $\xi(\xi>\eta)$;

\footnotetext{
${ }^{5}$ Inaba considered both the irreversible case (young $\rightarrow$ married $\rightarrow$ divorced), with reproduction via first marriage (Inaba 1996) and a reversible case, with iterative marriage (1993). We also invite the reader to refer to his paper for the relevant mathematical literature on multistate stable population theory.
} 
ii) the number of adult women with zero duration of permanence in the adult state and chronological age at adulthood $\eta$ at time $t$, is given by the number of individuals aged $\eta$ at time $t$ who became adult;

iii) the number of individuals with zero marriage duration who entered the adult state at age $\eta$ and married at $\xi$ is given by the number of marriages of $p_{1}(c, t ; \eta)$ individuals at the age $\xi=\eta+c$

Finally, a set of prescribed initial distributions has to be assigned:

$$
p_{0}(a, 0)=H_{0}(a) \quad p_{1}(c, 0 ; \eta)=H_{1}(c, \eta) \quad p_{1}(\tau, 0 ; \eta, \xi)=H_{2}(\tau ; \eta, \xi) \quad p_{3}(a, 0)=H_{3}(a)
$$

We postulated that the fertility rates depend not only on the duration of marriage but also on the age at marriage, as in Inaba (1996), and on the age of transition to adulthood. The latter appears to be a reasonable point: in general, other things being equal, married individuals characterized by the same marriage duration and by the same age at marriage may be expected to have different fertility if they entered the adult state sooner. This since they probably experienced different life histories - mostly in terms of educational attainment and job experiences - than those who entered the adult state at a later age.

The mathematical treatment of the model (1)-(2)-(3) is a tedious but straightforward task, similar to what Inaba (1996) did for the three-state model. Since we are not interested here in the mathematical aspects, we omit most of the technical details (see Manfredi and Billari 1997), and simply recall the most relevant steps of the solution process. As a first step it is possible to show that the overall age distribution $n(a, t)=\sum_{i=0}^{3} p_{i}(a, t)$ satisfies a traditional Von Foerster partial differential equation (PDE). This makes it possible to reformulate the problem into a Lotka-type renewal model in the (chronological) age-time domain, governed by a traditional integral equation for births. This enables us to show the existence of an asymptotic stable behaviour in Lotka's sense, which is characterised by stable exponential growth over time of $B(t)$ when the net reproduction rate (NRR), $R_{0}$, of the population is greater than one and, 
vice-versa, by stable exponential decay when $R_{0}<1$. The existence of a stable distribution with respect to age and stages of life is easily proved by expressing the formal solutions of the equations (1) for $t$ large 'enough' (where 'enough' means that the terms depending on initial conditions vanish), in terms of the overall density $n(a, t)$. It holds that:

$$
\begin{aligned}
& p_{0}(a, t)=B(t-a) l(a) V(a)=n(a, t) V(a) \\
& p_{1}(c, t ; \eta)=n(a, t) V(\eta) v(\eta) \Lambda_{\eta}(c) \\
& p_{2}(\tau, t ; \eta, \xi)=n(a, t)\left[V(\eta) v(\eta) \Lambda_{\eta}(\xi) \lambda_{\eta}(\xi, \eta) \Delta_{\xi}(\tau)\right]
\end{aligned}
$$

The relations in (4) express the population densities in the states $0,1,2$ in terms of the total population $n(a, t)^{6}$ and of the following generalised survival functions:

$$
\begin{aligned}
& l(a)=\exp \left(-\int_{0}^{a} \mu(u) d u\right) \\
& V(a)=\exp \left(-\int_{0}^{a} v(u) d u\right) \\
& \Lambda_{\eta}(c)=\exp \left(-\int_{\eta}^{\eta+c} \lambda(u ; \eta) d u\right)=\exp \left(-\int_{0}^{c} \lambda(\eta+s ; \eta) d s\right) \\
& \Delta_{\xi}(\tau)=\exp \left(-\int_{0}^{\tau} \delta(u ; \xi) d u\right)
\end{aligned}
$$

where

i) $l(a)$ is the 'survival to natural mortality' function (the probability that a new-born woman survives to age $a$ );

ii) $V(a)$ is the 'survival to adulthood' function (the probability that a newborn woman does not enter adulthood before age $a$ in absence of mortality);

iii) $\Lambda_{\eta}(c)$ is the (conditional) 'survival to marriage' function (the probability that a woman who entered the adult state at age $\eta$ is still unmarried at age $a=\eta+c)$;

iv) $\Delta_{\eta}(c)$ the survival function to divorce until age $a=\tau+\xi$ for a woman married at age $\xi$. Suitable integrations on the quantities (4) lead to:

\footnotetext{
${ }^{6}$ They represent the formal solutions to the corresponding PDE's (6) for $t>a$. Such solutions are "formally" derived by integration along characteristics of the given PDE's, but they can be derived in a totally direct way by sequentially applying the survival functions (5).
} 


$$
\begin{array}{ll}
p_{0}(a, t)=n(a, t) V(a) & \\
p_{1}(a, t)=n(a, t) \Psi(a) ; \quad \Psi(a)=\int_{0}^{a} V(\eta) v(\eta) \Lambda_{\eta}(a-\eta) d \eta \\
p_{2}(a, t)=n(a, t) \Gamma(a) ; \quad \Gamma(a)=\int_{0}^{a} \int_{0}^{\xi}\left[V(\eta) v(\eta) \Lambda_{\eta}(\xi) \lambda_{\eta}(\xi, \eta) \Delta_{\xi}(a-\xi)\right] d \eta d \xi
\end{array}
$$

The relations (6) show that when the total population $n(a, t)$ reaches its stable age distribution, it will automatically have a stable distribution by stages of life as well.

\subsection{Indices of reproduction (and structure)}

In what follows, we provide some formulas for the Net Reproduction Rate (NRR) and the Total Fertility Rate (TFR) by generalising Inaba's (1996) work to the present four-state framework. As concerns the NRR, the following equivalent definitions hold:

$$
\begin{aligned}
& R_{0}=\int_{0}^{\beta} \int_{\eta}^{\beta} \int_{0}^{\beta-\xi} m(\tau ; \xi, \eta) l(\xi+\tau) V(\eta) v(\eta) \Lambda_{\eta}(\xi) \lambda(\xi, \eta) \Delta_{\xi}(\tau) d \tau d \xi d \eta \\
& =\int_{0}^{\beta} \int_{0}^{\beta-\tau} \int_{0}^{\xi} m(\tau ; \xi, \eta) l(\xi+\tau) V(\eta) v(\eta) \Lambda_{\eta}(\xi) \lambda(\xi, \eta) \Delta_{\xi}(\tau) d \eta d \xi d \tau \\
& =\int_{0}^{\beta} \int_{0}^{\xi} \int_{0}^{\beta-\xi} m(\tau ; \xi, \eta) l(\xi+\tau) V(\eta) v(\eta) \Lambda_{\eta}(\xi) \lambda(\xi, \eta) \Delta_{\xi}(\tau) d \xi d \eta d \tau
\end{aligned}
$$

The change of variable from the 'old' set $(\eta, \xi, \tau)$ to the 'new' one $(\eta, \xi, a=\xi+\tau)$, leads to the following alternative definition:

$$
R_{0}=\int_{0}^{\beta} l(a)\left[\int_{0}^{a} V(\eta) v(\eta)\left(\int_{\eta}^{a} \Lambda_{\eta}(\xi) \lambda(\xi, \eta) \Delta_{\xi}(\tau) m(\tau ; \xi, \eta) d \xi\right) d \eta\right] d a
$$

Equation (8) suggests the following definition for the age-specific fertility rates:

$$
m^{*}(a)=\int_{0}^{a} V(\eta) v(\eta)\left(\int_{\eta}^{a} \Lambda_{\eta}(\xi) \lambda(\xi, \eta) \Delta_{\xi}(\tau) m(\tau ; \xi, \eta) d \xi\right) d \eta
$$

Definition (9) relates the traditional age-specific fertility rate with the duration-specific fertility rates. If we explicitly neglect mortality, the following definition for the TFR (total number of offspring produced by a woman during her 'effective' fertile age span, the latter given by the portion of the her fertile age span actually spent within the married state) arises:

$$
T F R=\int_{0}^{\beta} \int_{\eta}^{\beta} \int_{0}^{\beta-\xi} m(\tau ; \xi, \eta) V(\eta) v(\eta) \Lambda_{\eta}(\xi) \lambda(\xi, \eta) \Delta_{\xi}(\tau) d \tau d \xi d \eta
$$

By stressing the sequential nature of the stages of life, we can write: 


$$
\begin{aligned}
& T F R=\int_{0}^{\beta} V(\eta) v(\eta)\left[\int_{\eta}^{\beta} \Lambda_{\eta}(\xi) \lambda(\xi, \eta) T(\xi, \eta) d \xi\right] d \eta= \\
& =\int_{0}^{\beta} T^{*}(\eta) V(\eta) v(\eta) d \eta
\end{aligned}
$$

where:

$$
T(\xi, \eta)=\int_{0}^{\beta-\xi} m(\tau ; \xi, \eta) \Delta_{\xi}(\tau) d \tau \quad ; \quad T^{*}(\eta)=\int_{\eta}^{\beta} \Lambda_{\eta}(\xi) \lambda(\xi, \eta) T(\xi, \eta) d \xi
$$

The quantity $T(\xi, \eta)$ is the conditional TFR of those women who became adult at the age of $\eta$ and married at the age of $\xi$ in presence of the risk of marriage dissolution. As concerns the quantity $T^{*}(\eta)$ we can write:

$$
T^{*}(\eta)=P E M(\eta) \cdot \int_{\eta}^{\beta} T(\xi, \eta) \Phi_{\eta}(\xi) d \xi
$$

where:

$$
\Phi_{\eta}(\xi)=\frac{\Lambda_{\eta}(\xi) \lambda(\xi, \eta)}{\int_{\eta}^{\beta} \Lambda_{\eta}(\xi) \lambda(\xi, \eta) d \xi} \quad 0 \leq \eta \leq \beta ; \eta \leq \xi \leq \beta
$$

represents the conditional (normalised) density function of marriage at age $\xi$ for women who entered the adult state at age $\eta$ and married before the end of the fertile age span, and:

$$
\operatorname{PEM}(\eta)=\int_{\eta}^{\beta} \Lambda_{\eta}(\xi) \lambda(\xi, \eta) d \xi=1-\Lambda_{\eta}(\beta)
$$

is the (conditional) proportion of ever-married (PEM) women (to be precise: those who married before age $\beta$ ) among those women who entered the adult state at the age of $\eta$. By further defining:

$$
W(\eta)=\frac{V(\eta) v(\eta)}{1-V(\beta)} \quad ; \quad \int_{0}^{\beta} V(\eta) v(\eta) d \eta=1-V(\beta)=P E A(\beta)
$$

where $W(\eta)$ is the normalised probability density of transition to adulthood (truncated at the end of the fertile age span) and $\operatorname{PEA}(\beta)$ is the 'proportion ever adult' at the end of the fertile period, we can write the TFR as:

$$
T F R=P E A(\beta) \int_{0}^{\beta} T^{*}(\eta) W(\eta) d \eta
$$


By introducing (13) into (17) and assuming, quite reasonably, that $P E A(\beta)=1$, we obtain:

$$
T F R=\int_{0}^{\beta} \operatorname{PEM}(\eta) W(\eta)\left[\int_{\eta}^{\beta} T(\xi, \eta) \Phi_{\eta}(\xi) d \xi\right] d \eta=\int_{0}^{\beta} \operatorname{PEM}(\eta) W(\eta) \bar{T}(\eta) d \eta
$$

where $\bar{T}(\eta)$ is the TFR (averaged with respect to the age at marriage) of women who entered into adulthood at age $\eta$ and married before the end of the fertile age span. $\bar{T}(\eta)$ is a conditional average TFR (conditional on having entered into adulthood at age $\eta$ ). It seems useful, at this stage, to distinguish two main sub-cases:

a) the fertility rates do not depend on the age of entrance into the adult state, i.e.: $m=m(\tau ; \xi)$. We call this case the 'pure delayer' case, in that the role of the intermediate state of adulthood does not affect the fertility behaviour of the (married) population: its effects are essentially those of delaying fertility by delaying marriage.

b) a more general case in which $m=m(\tau ; \xi, \eta)$, so that in addition to the 'delayer' effect, true fertility effects arise due to the process of transition to adulthood.

This latter distinction will be used in this paper when we deal with empirical applications.

\section{SOME NOTEWORTHY SPECIFICATIONS OF THE GENERAL MODEL}

In this section we analytically develop two interesting specifications of the general model. The first one - with transition rates independent on age - will help us to gain a better understanding of the model from the mathematical point of view. The second one will provide a linear approximation, which will become useful for the application to real data.

\section{$\underline{3.1 \text { Transition rates independent of age }}$}

Here we consider the special case of age-independent rates. Although oversimplified, this case is useful from several standpoints: it helps to clarify the role of 'sequential' stages within classical stable population theory, and it represents an instance of explicit parameterisation 
within our general framework. Let $p_{i}(t)(\mathrm{i}=0,1,2,3)$ denote the total numbers of young, adult, married, and 'residual' individuals, respectively, at time $t$. Further, let $\mu_{i}$ 's be the (ageindependent) state-specific death rates per unit of time (p.u.t. since now on), $v$ the (ageindependent) rate of transition to adulthood p.u.t., $\lambda$ the marriage rate p.u.t of adult individuals, $\delta$ the total rate of marriage dissolution, and $m$ the marital fertility rate. When all the rates in the general model (1) are constant, the general PDE system (1) reduces to the following system of ordinary linear differential equations (ODE since now on):

$$
\begin{aligned}
& \dot{p}_{0}(t)=m p_{2}(t)-\left(\mu_{0}+v\right) p_{0}(t) \\
& \dot{p}_{1}(t)=v p_{0}(t)-\left(\mu_{1}+\lambda\right) p_{1}(t) \\
& \dot{p}_{2}(t)=\lambda p_{1}(t)-\left(\mu_{2}+\delta\right) p_{2}(t) \\
& \dot{p}_{3}(t)=\delta p_{2}(t)-\mu_{3} p_{3}(t)
\end{aligned}
$$

The system $(19)^{7}$ can be represented compactly as $\dot{P}(t)=M P(t)$, where $M$ is the matrix:

$$
M=\left(\begin{array}{cccc}
-\left(\mu_{0}+v\right) & 0 & m & 0 \\
v & -\left(\mu_{1}+\lambda\right) & 0 & 0 \\
0 & \lambda & -\left(\mu_{2}+\delta\right) & 0 \\
0 & 0 & \delta & -\mu_{3}
\end{array}\right)
$$

The demographically relevant features of system (19) are easily inferred from those of the matrix $M$, which is a Metzler matrix. So, in particular, $M$ has a unique dominant eigenvalue $\mathrm{K}_{0}$, to which belongs a demographically meaningful (i.e.: non-negative) eigenvector. Furthermore, all remaining eigenvalues of $M$ have a real part which is less than $K_{0}$.

It is easy to see that the sign of the dominant eigenvalue $K_{0}$, which corresponds to Lotka's intrinsic rate of growth of the population and hence determines its long-term behaviour, depends only on the sign of the coefficient of the known term of the characteristic polynomial $\mathrm{P}(\mathrm{K})$ :

$$
P(K)=\left(K+\mu_{3}\right)\left(K^{3}+a K^{2}+b K+c\right)=0
$$

\footnotetext{
${ }^{7}$ Systems such as (1) are very common in population biology and population dynamics of infectious diseases. See, for instance, Anderson and May (1991).
} 
where:

$$
\begin{aligned}
& a=\left(\mu_{0}+v\right)+\left(\mu_{1}+\lambda\right)+\left(\mu_{2}+\delta\right)>0 \\
& b=\left(\mu_{0}+v\right)\left(\mu_{1}+\lambda\right)+\left(\mu_{0}+v\right)\left(\mu_{2}+\delta\right)+\left(\mu_{1}+\lambda\right)\left(\mu_{2}+\delta\right)>0 \\
& c=\left(\mu_{0}+v\right)\left(\mu_{1}+\lambda\right)\left(\mu_{2}+\delta\right)-m v \lambda
\end{aligned}
$$

Hence, $\mathrm{K}_{0}$ will be positive or negative (i.e. we will have stable exponential growth rather than stable exponential decay) depending on whether:

$$
m v \lambda-\left(\mu_{0}+v\right)\left(\mu_{1}+\lambda\right)\left(\mu_{2}+\delta\right) \frac{\geq}{\leq} 0
$$

The threshold condition (22) can be represented as:

$$
R_{0}=\frac{m}{\mu_{2}+\delta} \cdot \frac{v}{\mu_{0}+v} \cdot \frac{\lambda}{\mu_{1}+\lambda}>1
$$

where $R_{0}$ defines the NRR of the population for this unstructured case (see Manfredi and Billari 1997). This last result clearly shows that, in the presence of below replacement fertility, no policy aimed at bringing fertility up to the 'zero growth level' that is based solely on a reduction of the age at marriage and/or age of transition to adulthood can be completely successful. The other way round, acting suitably on such parameters can prove to be an effective policy for bringing a population experiencing stable growth down to a stationary level. An important example could be, for instance, a policy of systematically increasing literacy and education in developing countries.

The long-term age structure by state which is implicit in model (19) can be determined analytically (Manfredi and Billari, 1997). In the standard case, in which mortality is stateindependent (i.e.: $\mu_{i}=\mu$ for all $i$ ), the population weights $w_{i}(a)=p_{i}(a, t) / n(a, t)$ that emerge in the long-term stable regime are given by 


$$
\begin{aligned}
& w_{0}(a)=\exp (-v a) \\
& w_{1}(a)=\frac{v}{v-\lambda}[\exp (-\lambda a)-\exp (-v a)] \\
& w_{2}(a)=\frac{v \lambda}{v-\lambda} \exp (-\delta a)\left\{\frac{1-\exp [-(\lambda-\delta) a]}{\lambda-\delta}-\frac{1-\exp [-(v-\delta) a]}{v-\delta}\right\} \\
& w_{3}(a)=1-\sum_{0}^{2} w_{i}(a)
\end{aligned}
$$

A numerical example of the stable population distribution according to (24), on a yearly time scale, with the rates fixed at $v=1 / 19, \lambda=1 / 11, \delta=1 / 36$, respectively (corresponding to an average age of transition to adulthood of 19 years, an average duration of permanence in the adult state of 11 years, and an average duration of marriage of 36 years $\left.^{8}\right)$, is given in Figure 2.

In Figure 3, we plotted $R_{0}$ as a function of the fertility rate $m$ and the rate of transition to adulthood $v$, keeping the other variables constant at the values given before (with a common mortality rate of $1 / 100$ ). We see clearly that, if $R_{0}$ is linearly dependent on fertility, a rise in rates of transition to adulthood also has an impact, which is however marginally decreasing.

\section{[Figures 2 and 3 about here]}

\subsection{A linear approximation}

By resorting to some linear approximations we derive here some nice formulas relating the impact of changes in the mean age of transition to adulthood and the TFR. Let us consider the 'pure delayer' case (section 2.3), in which the fertility rates of married women do not depend on their age of entry into adulthood. In this case we have, from (12a):

$$
T(\xi, \eta)=\int_{0}^{\beta-\xi} m(\tau ; \xi) \Delta_{\xi}(\tau) d \tau=T(\xi)
$$

Let us now assume, following again Inaba (1996), a Henry-type (Henry, 1976) linear relation between the conditional TFR of women married at age $\xi, T(\xi)$ and the age at marriage:

$$
T(\xi)=U-V \xi+R(\xi) \quad U>0, V>0
$$

\footnotetext{
${ }^{8}$ These values are quite reasonable for the Italian case.
} 
where $\mathrm{R}$ is a remainder. Neglecting the reminder, we get from the relation (18) the approximated relation:

$$
T F R=U \int_{0}^{\beta} P E M(\eta) W(\eta) d \eta-V \int_{0}^{\beta} P E M(\eta) W(\eta) \bar{\xi}(\eta) d \eta
$$

where $\bar{\xi}(\eta)$ is the average age at marriage of the women who entered the adult state at the age of $\eta$ and married before the end of the fertile age span. Let us introduce the quantities:

$$
B=\int_{0}^{\beta} P E M(\eta) W(\eta) d \eta \quad W *(\eta)=\frac{P E M(\eta) W(\eta)}{B} \quad 0 \leq \eta \leq \beta
$$

respectively, defining the 'average PEM', i.e. the average value of the conditional (on the age of entrance into adulthood) proportion ever married and the probability density function of transition to adulthood at age $\eta$ for women who married before the end of the fertile age span. Thanks to $(28 \mathrm{a}, \mathrm{b})$ we write $(27)$ as:

$$
T F R \cong B\left\{U-V \int_{0}^{\beta \bar{\xi}}(\eta) W *(\eta) d \eta\right\}=B\{U-V \bar{\xi}\}
$$

In (29), the overall TFR is factored as the product of the average PEM times the value of the Henry relationship evaluated in correspondence to the age at marriage $\bar{\xi}$ (averaged on the density of transition to adulthood) of the women married before the end of the fertile age span. If we assume $\bar{\xi}(\eta)$ to be linear, i.e.:

$$
\bar{\xi}(\eta)=\bar{\xi}\left(\eta_{A}\right)+q\left(\eta-\eta_{A}\right)=p+q \eta \quad \eta_{A} \leq \eta \leq \beta
$$

where $\eta_{A}$ is the lower bound in the possible ages of transition to adulthood and $\bar{\xi}\left(\eta_{A}\right)$ the corresponding average age at marriage, (29) leads to':

$$
T F R=B\left\{U-V \int_{0}^{\beta}[p+q \eta] W^{*}(\eta) d \eta\right\}=B\{U-p V-q V \bar{\eta}\}
$$

Formula (31) relates the TFR to the average PEM and the average age at adulthood and could therefore be used to roughly estimate the 'pure delayer' effects of an increase in the average age $\bar{\eta}$ of transition to adulthood of women married before the end of their fertile age span on the

\footnotetext{
${ }^{9}$ See Manfredi and Billari (1997) for further details.
} 
TFR. This operation, when empirically justifiable, requires merely that the parameters $V$ and $q$ are estimated.

\section{EMPIRICAL APPLICATIONS: DATA FROM THE ITALIAN CASE}

In the following two subsections, we shall discuss some distinct applications based on our mathematical model, both aimed at discussing the impact of changes in adulthood attainment. First, we will perform some macrosimulation on a discrete-time version of the model. Then, we will use the linear approximation. We first give some brief information on the data we use. To estimate rates of adulthood attainment, union formation, and fertility, we use individual data from the 1995/96 Italian Fertility and Family Survey (De Sandre et al., 1996), a retrospective survey of Italian men and women born between 1946 and 1975. The survey contains eventhistory information on the month and the year in which people left their parental home, left full time education, entered the first union, bore children, and other demographic events. As we are dealing with a one-sex model, we only use female data. The remaining data, which are not available from the FFS (mortality rates, separation and divorce rates, and rates of transition to widowhood) come from official statistics at the aggregate level (Istat (1996)).

The event of becoming an adult has been defined here as the first occurring event among the following three: i) leaving formal education, ii) leaving the parental home, or iii) attaining the age of 35 . We assume, however, that it is not possible to become an adult before the age of 15 , among other reasons because the lowest legal age at marriage is 16 . Hence, transition events occurring before the $15^{\text {th }}$ or after the $35^{\text {th }}$ birthday have been assigned to the corresponding birthday.

4.1 A multistate stable population experiment: simulating the macro-impact of a delayed rransition to adulthood 
In this section we illustrate a set of long-term (stable) macro-simulations ${ }^{10}$. Our aim is to evaluate the long-term and structural consequences arising from a 'pure delay' effect in the transition to adulthood, other things being constant. This means that delayed adulthood attainment can have an impact only via delaying marriages, and that marital fertility rates do not depend on the age at which young people become adult. For this reason, we split the sample of women from the FFS survey into two broad cohorts (the 'old', born 1946-1960 and the 'young', born 1961-1975) ${ }^{11}$. The first cohort provides a 'benchmark' stable scenario, whereas the second, being characterised by a delayed pattern of transition to adulthood (Fig. 4), is used to provide an 'alternative' scenario. The average age at adulthood is 17.8 and 21.3 , respectively. Our general idea is to evaluate the effect of a delayed transition to adulthood with a sort of 'comparative static' approach, by placing the stable distributions that arise from both scenarios side by side.

The only difference between the benchmark and the alternative scenario is thus represented by the patterns of adulthood attainment. All other schedules (rates of transition from the adult state to the married state, fertility rates of the married women, rates of transition from the married state to the residual one, and the mortality rates) are kept equal. In particular, we estimated from the old cohort $^{12}$ : transition rates from the adult to the married states (Fig. 5), age-specific marital fertility rates (Fig. 5), and marriage duration-specific marital fertility rates (Fig. 6). We also estimated marital fertility rates depending jointly on age and marriage duration, where the latter has only four categories for reasons of stability in estimates (Fig. 7).

\section{[Figures 4-7 about here]}

As the initial age structure in our simulations we used the age distribution observed in the 1991 Census of the Italian population. The figures observed for 1991 were then subdivided with

\footnotetext{
${ }^{10}$ Wolf (1988) outlines the application of a multistate life table which in fact contains all the necessary tools to fully exploit the mathematical model. He also emphasises the demanding data needs of his approach.

${ }^{11}$ The same cohort definition was used, for instance, by Geremei (1999).
} 
respect to the four states, according to the corresponding figures observed in the stationary population.

Some information on the benchmark scenario comes from the stationary population, corresponding to the overall set of transition rates estimated for the benchmark scenario - that is, the population distribution in the case of zero growth. In this case, the distribution by state of the overall population is: $21.4 \%$ in the young, $9.9 \%$ in the adult, $48.5 \%$ in the married, and $20.6 \%$ in the no longer married state. In terms of life expectancy, a woman lives on average 80 years, of which 17.1 are lived in the young, 7.9 in the adult, 38.5 in the married, and 16.5 in the residual state.

We now compare the benchmark and the alternative scenario with three different simulations that were run until the stable structure was achieved with the desired accuracy ${ }^{13}$. Our comments will be focused on the effect on age structure and on reproduction.

In the first simulation, marital fertility is dependent on age only, as presented in Figure 4. Figure 7 compares the resulting stable distributions, while some synthetic measures are reported in Table 1a. Here the delay in adulthood attainment has an extraordinary effect. For instance, the stable TFR goes from 1.6 to 1.23 , and the ratio of people aged 65 and over to people aged 20-64 goes from $159 \%$ to $293 \%$. The mean age of the population increases by more than five years, and also the dependency index is significantly affected. The reason is that, if marital fertility depends on age only, there can be no recuperation for childbearing purposes of the years 'lost' because of delayed transition to adulthood; this also has obvious consequences concerning population ageing.

Then, we take the opposite point of view and simulate the model when marital fertility depends on the duration of marriage only, as in Figure 6. In this case (Figure 9, Table 1b), the effect of

\footnotetext{
${ }^{12}$ Our estimates use person-years, in order to avoid censoring problems, and the linear integration hypothesis.

${ }^{13}$ The simulations were performed with a program written in Visual Basic for Excel by A. Valentini.
} 
delayed transition to adulthood is less significant. There are still some differences due to the biological limits of reproduction.

The third simulation is in between, uses more information, and is more useful as a representation of real situations. Marital fertility there depends both on age and duration of marriage, where the latter has been split into four categories (Figure 7). The results are displayed in Figure 10 and Table 1c. The stable TFR in this case goes from 1.74 to 1.56. Thus, the effect of a delay in adulthood attainment of an average of $31 / 2$ years is diminishing fertility of around 0.2 children. The impact on the stable structure is indeed important: the mean age of the population rises from 45.3 to 47.6 , and the ratio of people aged 65 and over to people aged 20-64 goes from $131 \%$ to $167 \%$. The latter results provide us with an important evaluation of the impact of delayed adulthood attainment.

[Figures 8-10 and Table 1 about here]

4.2 The impact of increases in the mean age at transition to adulthood on fertility: use of the linear approximation

In this section the mathematical relationships (26), (30), and (31), which were developed in subsection 3.2, are used to gain insights into the effect of changing tempo in adulthood attainment on the reproduction of the population. We only refer here to women aged 40 and over (born 1946-1955) in order to avoid censoring problems.

First of all we follow the approach of Inaba (1996) and use the linear approximation (26): $T(\xi)=U-V \xi$ (first proposed by Henry (1976)) between the number of children ever born and the age at first union. Though in principle very rough, this is essentially a local approximation. It has the merit of being both surprisingly accurate from the empirical point of view and mathematically manageable. It is thus possible to estimate via linear regression the relationship $T(\xi)=U-V \xi$ using our individual-level data. The results of a simple OLS regression are reported in Table 2. Notice that the $R^{2}$ values are computed on the conditional average values of 
the TFR computed for each age at union and not on the individual data (Fig. 11), so as to stress that aggregate-level data could be used for this approximation. With reproductive behaviour such as that exhibited by the cohort we considered, a one-year increase in the age at marriage would bring down the number of female children ever born ${ }^{14}$ by 0.0769 .

Second, to evaluate the effect of an increase in the age of attaining adulthood on the average age at marriage we adopt the linear approximation (30). This assumption is not common, but it permits us to remain close to the spirit of the Henry-type relationship (an approximate but possibly useful one ${ }^{15}$ ), and it seems to be well-justified by the available data (Table 2 and Fig. 12). Although very heuristic, the linear approximation seems to work quite well. It is worth noticing that a one-year increase in adulthood attainment provokes a 0.54 year increase in age at marriage (note that the estimated regression coefficient is, as expected, less than one) as women who attained adulthood later tend to have a shorter interval between adulthood attainment and entering a union) ${ }^{16}$. Finally, in order to obtain the approximate TFR, we computed the mean age at union and the proportion ever in union from our data. By combining the two linear relations, the expected decrease in the TFR for women who ever enter a union as a consequence of a one-year delay in adulthood attainment, given by the product $(B V q)$ (see (31)), is definitively equal to $0.041^{17}$.

A comparison between this result and the results obtained from the macrosimulation is of particular interest to us. Using the mean age at adulthood attainment of the former benchmark and alternative scenario (17.8 and 21.3 respectively), and by assuming constant parameters for the linear relationships estimated, we can evaluate the change in the TFR of ever-married

\footnotetext{
${ }^{14}$ Using Japanese data, Inaba (1996) computed an expected increase in the TFR of 0.11. Using data for younger Italian cohorts, Manfredi and Billari (1997) computed an expected increase in the TFR of 0.080.09 .

${ }^{15}$ The same assumption, though in the framework of a structural equations model, was used by Marini (1985).

${ }^{16}$ Using data on earlier Italian cohorts and the average age at attaining the highest educational level as a marker of attaining adulthood, Manfredi and Billari (1997) estimated that an increase of one year in women's age at adulthood would lead to an increase of about $0.4 / 0.5$ years in the age at marriage.
} 
women to be $0.041 \cdot(17.8-21.3)=-0.14$. If the PEM stays as high as in the benchmark scenario - a somewhat strong hypothesis, as it is $95 \%$ - the expected decrease in the total TFR is slightly less than -0.14 children per woman. This result is not far from the decrease we obtained in the simulation with marital fertility dependent on both age and marriage duration $(-0.18)$. This difference could be due to the fact that the simulation takes into account the effects on the PEM, at least the one for the reproductive ages, of delayed transition to adulthood. With the other two simulations, the values obtained were -0.08 when age has no influence and -0.37 when marriage duration has no influence.

The general impression is that the two linear approximations, with some supplementary hypotheses on the PEM, can be easy to compute and give significant results. They have the further advantage of require only limited data.

\section{[Figures 11-12 and Table 2 about here]}

\section{CONCLUSIONS AND DIRECTIONS FOR FUTURE RESEARCH}

This paper has been concerned with the macro-demographic consequences of delays in the transition to adulthood within a multistate stable population framework. We developed a particular multistate stable population model which is particularly suitable for the study of the 'Mediterranean pattern', and we discussed some analytical results such as the stable population structure. We showed that some interesting sub-cases - for instance the one with constant transition rates - can provide useful information on the mechanisms that affect the dynamics of population in such cases.

We then applied the model to the Italian case, estimating its parameters either from survey data or from official statistics. Some macrosimulations of the model allowed us to assess the impact of delayed adulthood on both reproduction and the structure of the population empirically. The

\footnotetext{
${ }^{17}$ This result substantially confirms the decrease of $0.03-0.04$ in the TFR computed with the same approach in a paper by Manfredi and Billari (1997).
} 
application of two simple linear approximations gave an easy-to-use tool for evaluating the impact on reproduction.

One must taken into account that the comparison of stable states is somewhat of a parable: it cannot be directly interpreted as a projection, but rather as a tool for the assessment of present trends. In the Italian case, for instance, a change in union formation or fertility patterns or immigration can change the picture we outlined. In general, our idea is that simulation of population models is particularly useful, but it is even more powerful when supplemented by some analytical support.

Much can still be done, in our opinion, on the theoretical side of the model. It seems promising to analytically discuss and apply more general multistate models, such as reversible models, i.e. models that embed the effects of remarriage, which can become highly relevant, and of the existence of different routes to marriage. It also appears desirable to move to more realistic two-sex frameworks, although this would really limit the possibility for analytical results. In addition, migration can be the issue of further generalisations. On the applied side, possible noteworthy effects of changing patterns of adulthood attainment on the management of pay-asyou go pension systems (such as the one common in Europe) seem to provide a further area of interest.

\section{REFERENCES}

Anderson R.M., and May R.M. (1991), Infectious diseases of humans: dynamics and control, Oxford: Oxford University Press.

Billari F.C. (1999), How to describe the transition to adulthood? The case of Italy, paper presented at the POLIS Project conference, Max Planck Institute for Human Development, Berlin.

Blossfeld H.-P., and De Rose A., (1992), Educational expansion and changes in entry into marriage and motherhood. The experience of Italian women, Genus, 48: 73-89. 
Coale A.J., and McNeil D.R. (1972), The distribution by age of the frequency of first marriage in a female cohort, Journal of the American Statistical Association, 67: 743-749.

Coleman J.S. (1990), Foundations of Social Theory, Cambridge MS and London: Belknap Press.

Corijn M. (1996), Transition into adulthood in Flanders. Results from the FFS, NIDI/CBGS Publications, Den Haag.

De Sandre P., Ongaro F., Rettaroli R. and Salvini S. (1997), Matrimonio e figli: tra rinvio e rinuncia, Bologna: il Mulino.

Geremei F. (1999), Union formation and chilbearing of Italian women. A LIPRO analysis on Italian FFS data, paper presented at the Joint ECE-EUROSTAT work session on Demographic Projections, Perugia.

Gilbert N., and Troitzsch K.G. (1999), Simulation for the Social Scientist, BuckinghamPhiladelphia: Open University Press,.

Henry L. (1976), Population: analysis and models, London: Edward Arnold.

Inaba H. (1988), A semigroup approach to the strong ergodic theorem of the multistate stable population process, Mathematical Population Studies, 11, 49-77.

Inaba H. (1993), A Mathematical Model for Human Population Reproduction via Iterative Marriage, Japanese Institute of Population Problems, WP n. 18, Tokio.

Inaba H. (1996), Human population reproduction via first marriage, Mathematical Population Studies, 5: 123-144.

Ledent J. (1988), Stable growth in native-dependent multistate population dynamics, Mathematical Population Studies, 1: 157-171.

Manfredi P. and Billari F. (1997), Transition into adulthood, marriage and timing of life in a stable population framework, Report n. 117, Dipartimento di Statistica e Matematica Applicata all’Economia, Università di Pisa, Pisa. 
Marini M.M. (1984), Age and sequencing norms in the transition to adulthood, Social Forces, 63: $229-44$.

Marini M.M. (1985), Determinants of the timing of adult role entry, Social Science Research, 14: $309-50$.

Reher D. (1998), Family ties in Western Europe: Persistent contrasts, Population and Development Review, 24, 203-234.

Rogers A. (1975), Multiregional demography, New York: Wiley.

Rogers, A. (1990), The multistate stable population model with immigration., Mathematical Population Studies, 2: 313-324.

Schoen R. (1988), Modelling multigroup populations, Plenum Press, New York.

Wolf D.A. (1988), The multistate life table with duration-dependence, Mathematical Population Studies, 1: 217-245. 


\section{LIST OF FIGURES}

Figure 1. Flow diagram of the general model.

Figure 2. State distributions by age in the case of transition rates independent of age (parameters: $v=1 / 19, \lambda=1 / 11, \delta=1 / 36$ ). 2a. Overall composition. 2b. Percentage distribution.

Figure 3. Net reproduction ratio as a function of fertility rates $(m)$ and of rates of transition to adulthood $(v)$, other rates constant.

Figure 4. Rates of transition to adulthood by age. Benchmark and alternative scenarios.

Figure 5. Transition rates from the adult to the married state and marital fertility rates (fertility dependent on age only).

Figure 6. Cumulated marital fertility rates (fertility dependent on marriage duration only).

Figure 7. Cumulated marital fertility rates (fertility dependent on age and marriage duration).

Figure 8. Age structure of the population (in millions) when marital fertility depends on age only.

Figure 9. Age structure of the population (in millions) when marital fertility depends on marriage duration only.

Figure 10. Age structure of the population (in millions) when marital fertility depends on age and marriage duration.

Figure 11. Total Fertility Rates conditional on given ages at first union and the linear approximation.

Figure 12. Age at first union conditional on given ages at adulthood attainment and the linear approximation. 


\section{LIST OF TABLES}

Table 1. Measures of synthesis in the benchmark and reference scenarios according to distinct hypotheses on fertility.

Table 2 Results from the OLS regressions between a) TFR and age at marriage, and b) age at marriage and age at adulthood. 


\section{FIGURES}

Figure 1. Flow diagram of the general model.

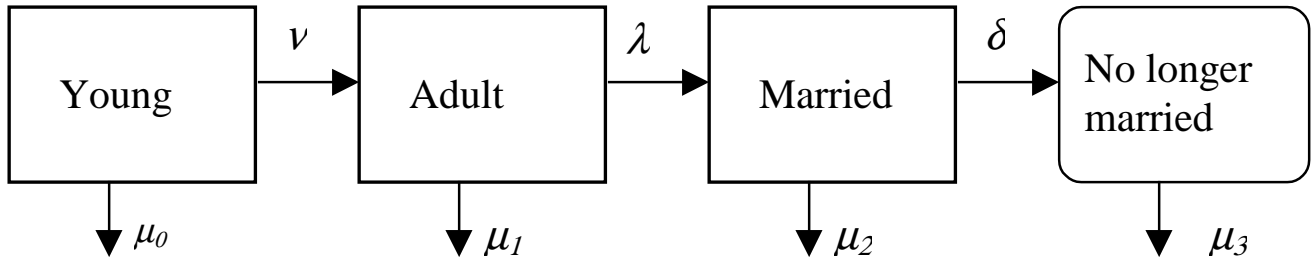


Figure 2. State distributions by age in the case of transition rates independent on age (parameters: $v=1 / 19, \lambda=1 / 11, \delta=1 / 36$ ).

2a. Overall composition

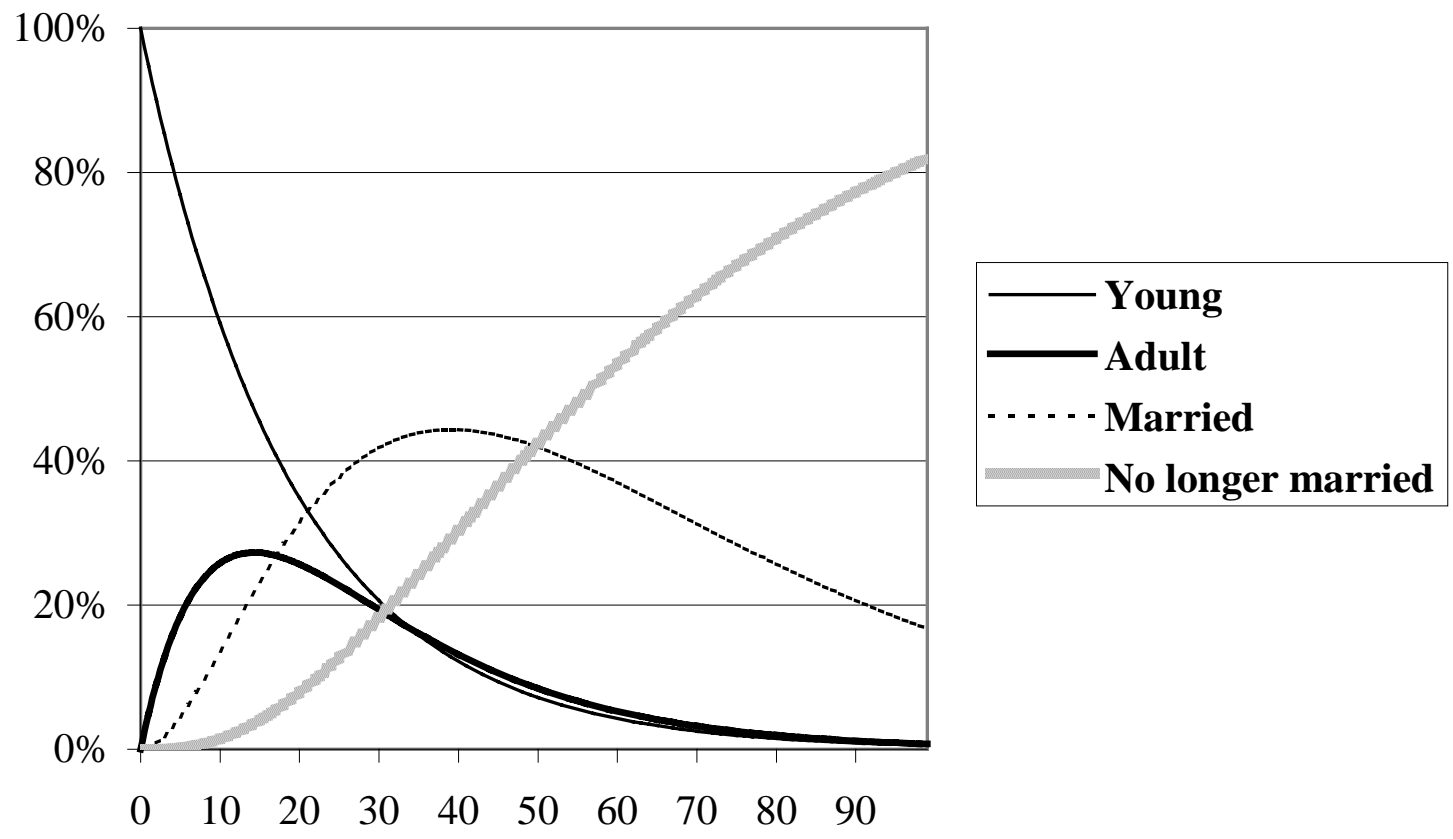

2b. Percentage distribution

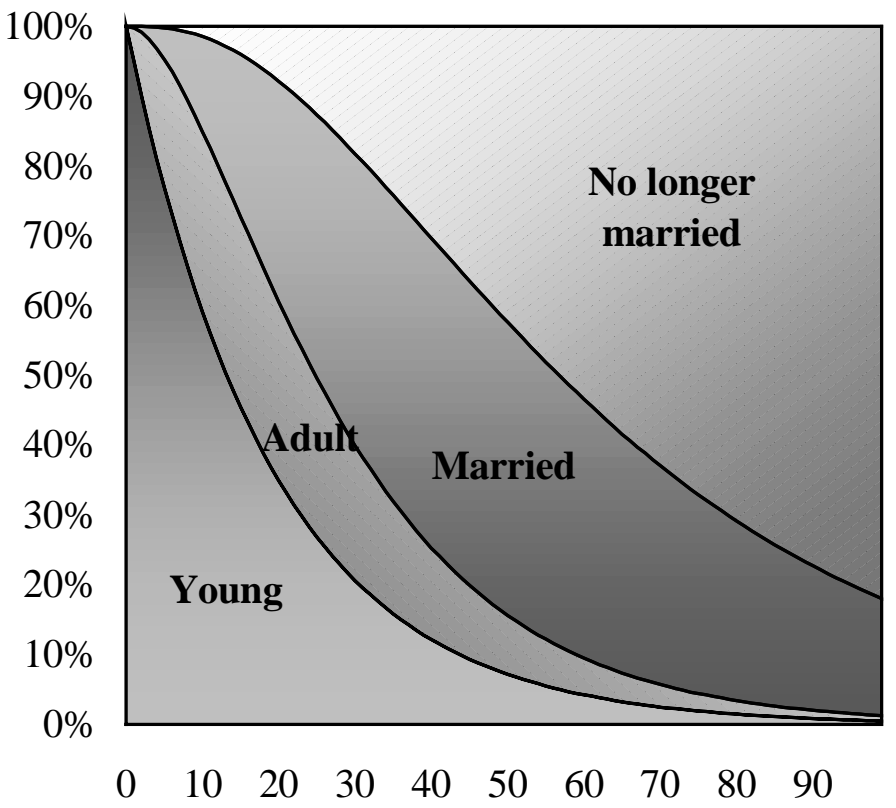


Figure 3. Net reproduction ratio as a function of fertility rates $(m)$ and of rates of transition to adulthood $(v)$, other rates constant.

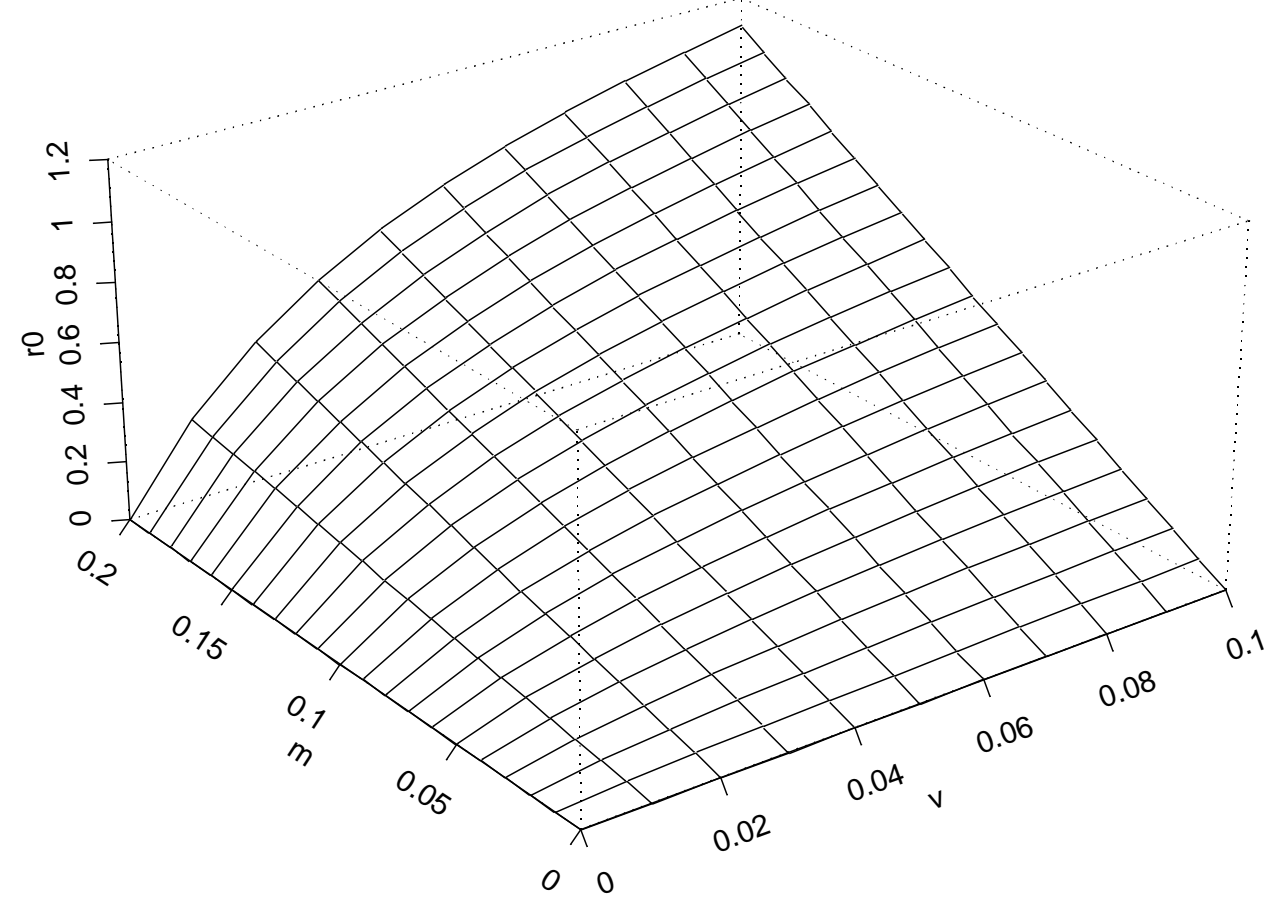


Figure 4. Rates of transition to adulthood by age. Benchmark and alternative scenarios.

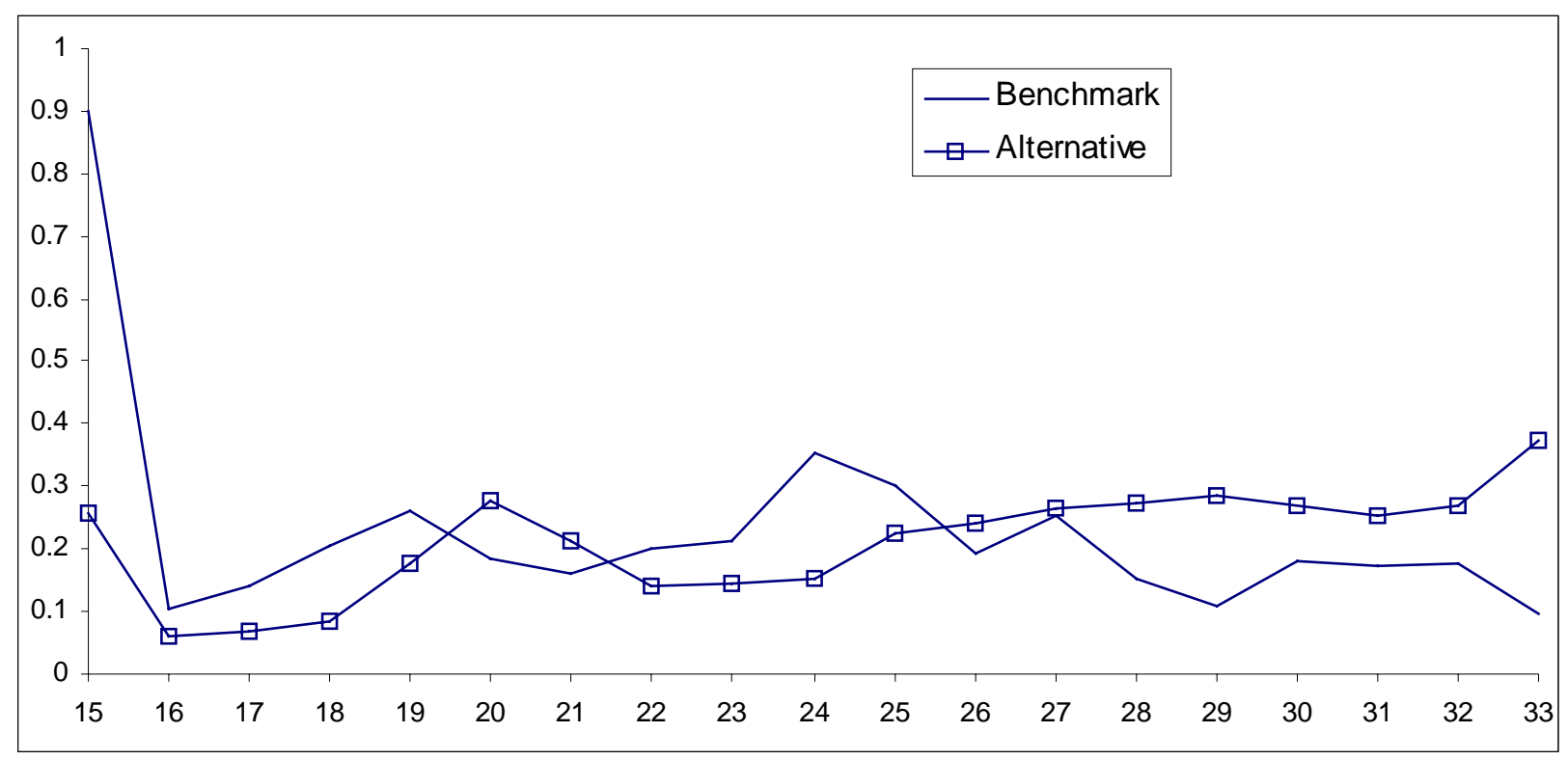

Figure 5. Transition rates from the adult to the married state and marital fertility rates (fertility dependent on age only).

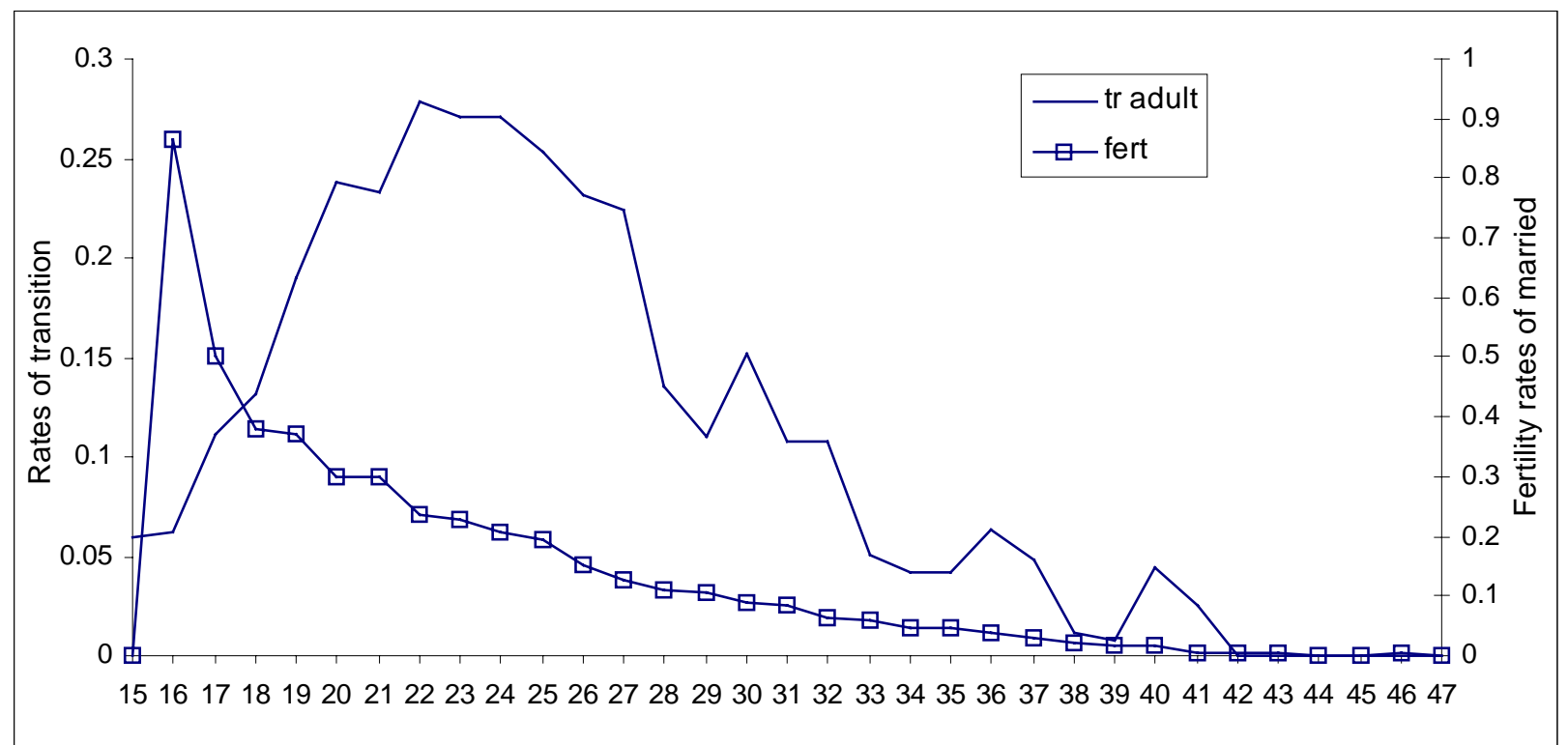


Figure 6. Cumulated marital fertility rates (fertility dependent on marriage duration only).

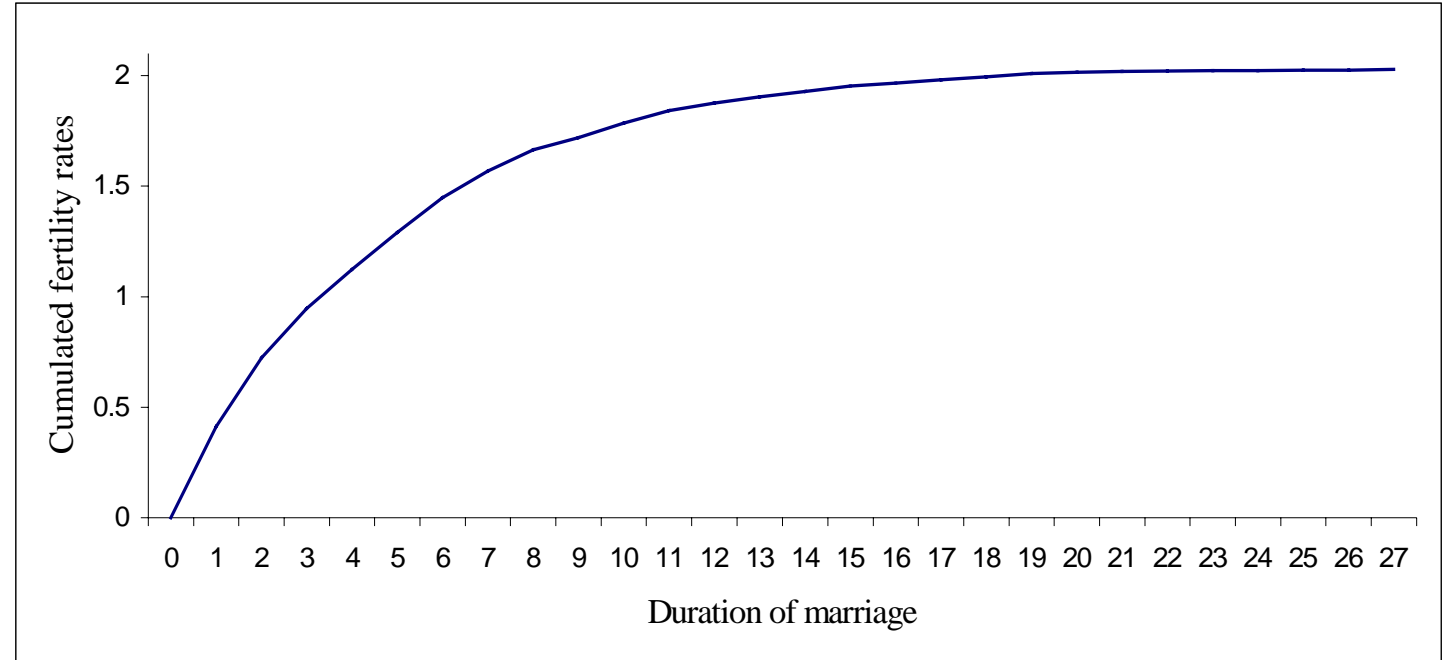

Figure 7. Cumulated marital fertility rates (fertility dependent on age and marriage duration).

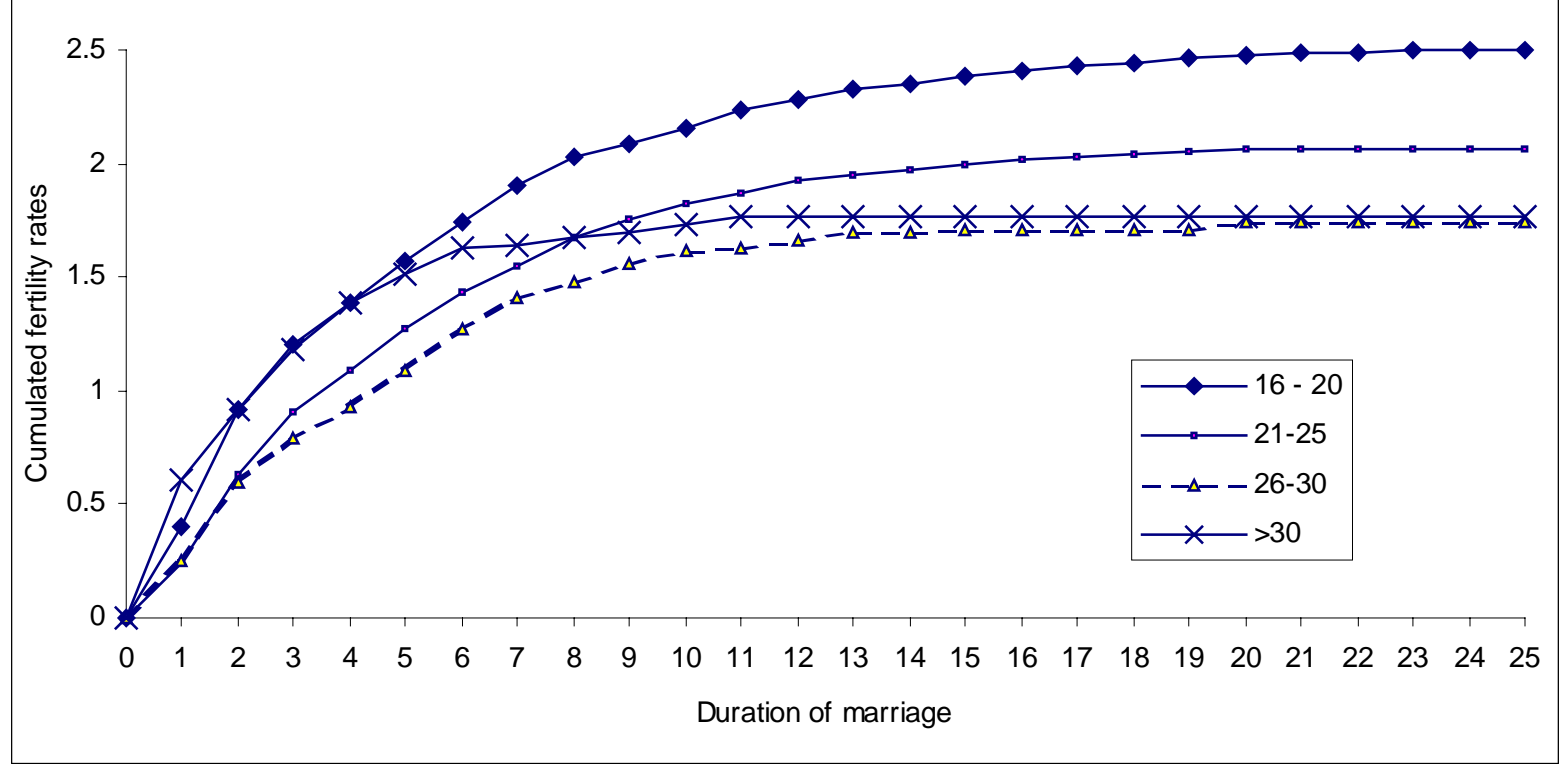


Figure 8. Age structure of the population (in millions) when marital fertility depends on age only.

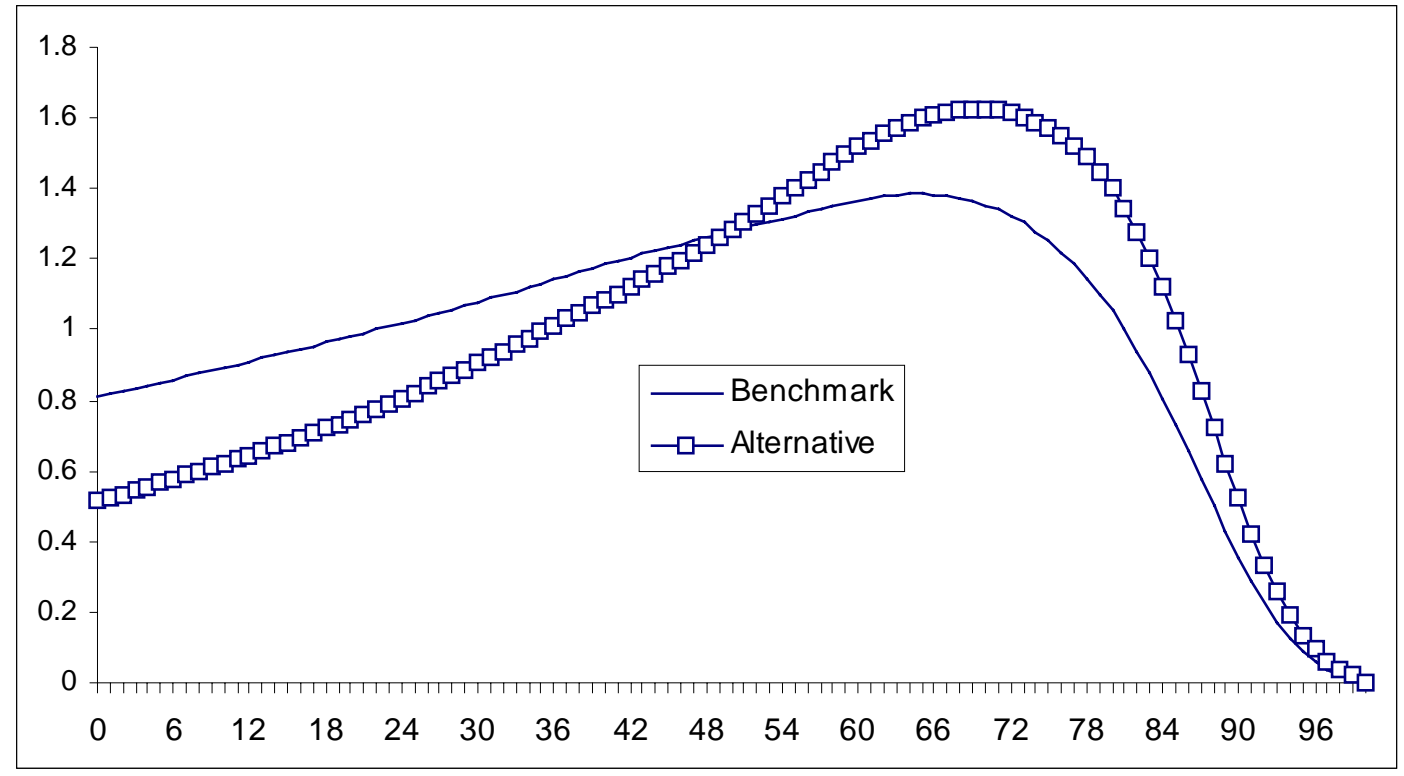

Figure 9. Age structure of the population (in millions) when marital fertility depends on marriage duration only.

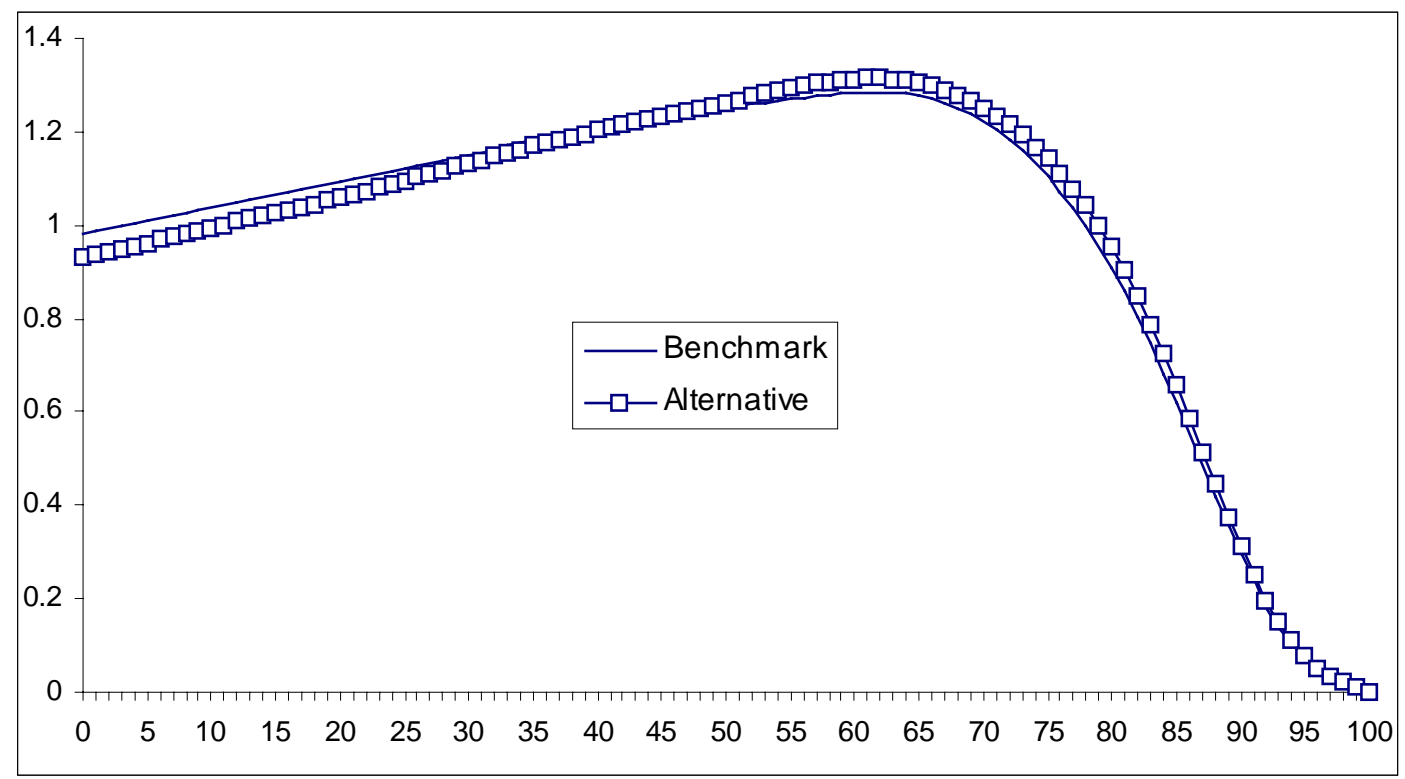


Figure 10. Age structure of the population (in millions) when marital fertility depends on age and marriage duration.

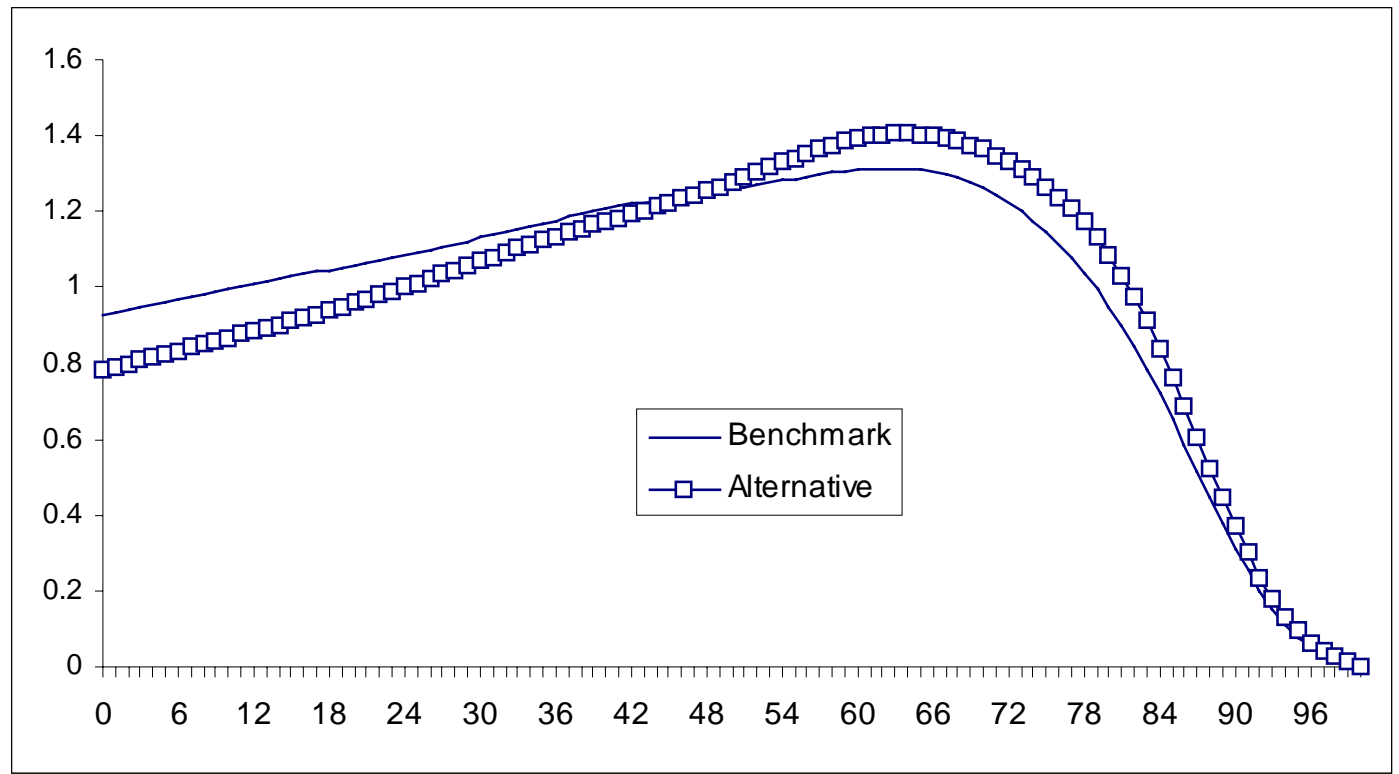


Figure 11. Total Fertility Rates conditional on given ages at first union and the linear approximation.

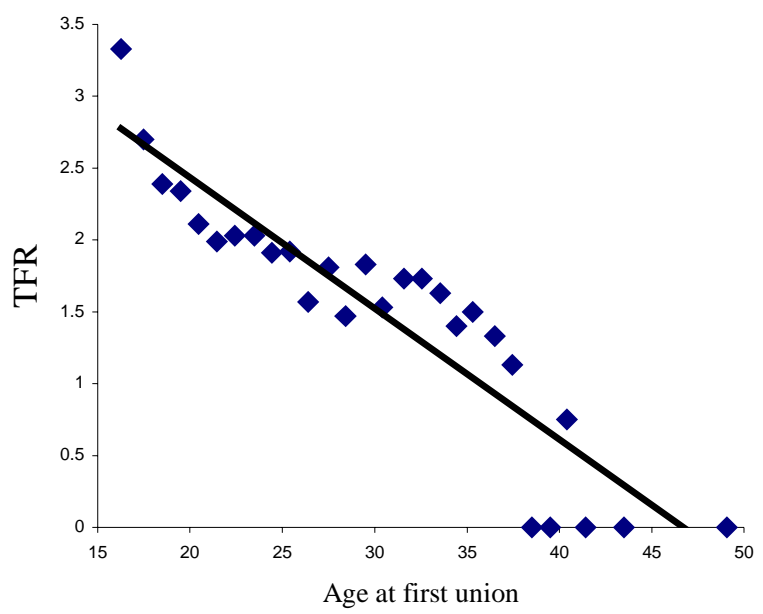

Figure 12. Age at first union conditional on given ages at adulthood attainment and the linear approximation.

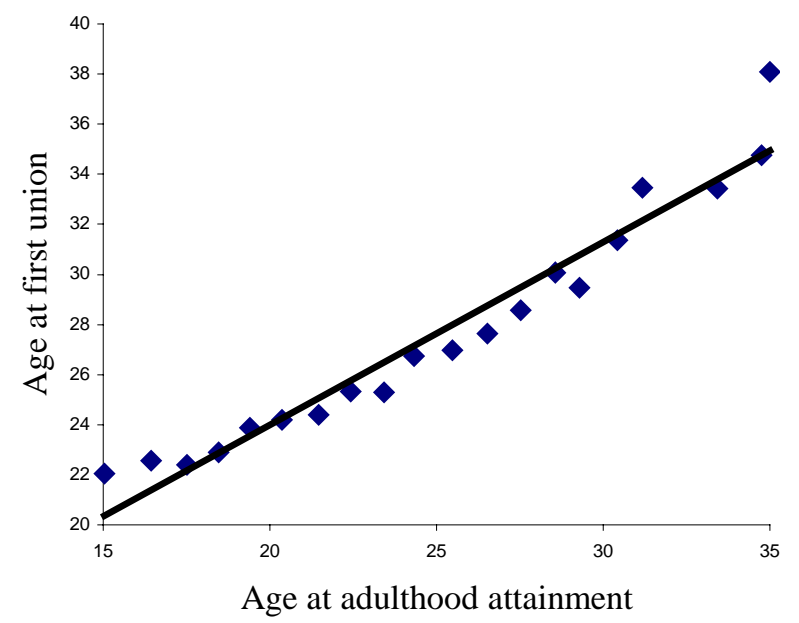


Table 1. Measures of synthesis in the benchmark and reference scenarios according to distinct hypotheses on fertility.

1a. Marital fertility dependent on age only

\begin{tabular}{|lcc|}
\hline & Benchmark & Alternative \\
\hline TFR & 1.60 & 1.23 \\
\hline Mean age & 47.12 & 52.54 \\
\hline Population 65+/Population 0-19 & 1.59 & 2.93 \\
\hline (Population 0-19 and 65+)/Population 20-64 & 0.86 & 0.94 \\
\hline
\end{tabular}

1b. Marital fertility dependent on marriage duration only

\begin{tabular}{|lcc|}
\hline & Benchmark & Alternative \\
\hline TFT & 1.79 & 1.71 \\
\hline Mean age & 44.57 & 45.31 \\
\hline Population 65+/Population 0-19 & 1.20 & 1.31 \\
\hline (Population 0-19 and 65+)/Population 20-64 & 0.83 & 0.84 \\
\hline
\end{tabular}

1c. Marital fertility dependent on age and marriage duration

\begin{tabular}{|lcc|}
\hline & Benchmark & Alternative \\
\hline TFT & 1.74 & 1.56 \\
\hline Mean age & 45.32 & 47.60 \\
\hline Population 65+/Population 0-19 & 1.31 & 1.67 \\
\hline (Population 0-19 and 65+)/Population 20-64 & 0.84 & 0.86 \\
\hline
\end{tabular}


Table 2 Results from the OLS regressions between a) TFR and age at marriage, and b) age at marriage and age at adulthood.

\begin{tabular}{|c|c|c|}
\hline Relation to be Estimated & $\begin{array}{c}\text { Parameter Estimates } \\
\text { (Standard Errors in parentheses) }\end{array}$ & $\mathrm{R}^{2}$ \\
\hline$T(\xi)=U-V \xi$ & $\mathrm{U}=3.8068(0.02880) \quad \mathrm{V}=0.07695(0.00120)$ & 0.7544 \\
\hline $\bar{\xi}(\eta)=p+q \eta$ & $\begin{array}{llll}\mathrm{p}=13.710 & (0.08266) & \mathrm{q}=0.5373 & (0.00458)\end{array}$ & 0.9088 \\
\hline
\end{tabular}

